
Sur seleccionados en el XIII encuentro de Editores, Montevideo, Uruguay 2008, para ser publicados por los países integrantes durante el año 2009.

\title{
Malformaciones Congénitas Asociadas a Agrotóxicos
}

\author{
S. BENÍTEZ-LEITE ${ }^{1}$, ML MACCHI ${ }^{1}$, M. ACOSTA ${ }^{2}$ \\ 1. Cátedra de Pediatría. Centro Materno Infantil (CMI). Facultad de Ciencias Médicas. UNA-Paraguay. \\ 2. Hospital Regional de Encarnación-Paraguay.
}

\begin{abstract}
Congenital Malformations Associated with Toxic Agricultural Chemicals

Introduction: Exposure to pesticides is a known risk for human health. This paper describes the relationship between parental exposure and congenital malformations in the newborn. Objective: To study the association between exposure to pesticides and congenital malformations in neonates born in the Regional Hospital of Encarnacion, in the Department of Itapua, Paraguay. Materials and Methods: A prospective case-controlled study carried out from March 2006 to February 2007. Cases included all newborns with congenital malformations, and controls were all healthy children of the same sex born immediately thereafter. Births outside the hospital were not counted. Exposure was considered to be any contact with agricultural chemicals, in addition to other known risk factors for congenital defects. Results: A total of 52 cases and 87 controls were analyzed. The average number of births each month was 216 . The significantly associated risk factors were: living near treated fields (OR 2.46, CI 95\% 1.09-5.57, p < 0.02), dwelling located less than $1 \mathrm{Km}$. (OR 2.66, CI 95\%; 1.19-5.97, p < 0.008), storage of pesticides in the home (OR 15.35, CI 95\%, 1.96701.63), $\mathrm{p}<0.03$ ), direct or accidental contact with pesticides (OR 3.19, CI 95\%, 0.97-11.4, p $<0.04$ ), and family history of malformation (OR 6.81, CI 5\%, 1.94- 30.56, p < 0.001). Other known risk factors for malformations did not show statistical significance. Conclusion: The results show an association between exposure to pesticides and congenital malformations. Further studies are required to confirm these findings.
\end{abstract}

(Key words: congenital malformations, agricultural chemicals, pesticide exposure).

Pediatr. (Asunción), Vol. 34; No 2; 2007

\section{RESUMEN}

Introducción: La exposición a plaguicidas es un riesgo reconocido para la salud humana. Se describe la relación entre la exposición de los padres y malformaciones congénitas en el neonato. Objetivo: Estudiar la asociación entre la exposición a pesticidas y malformaciones congénitas en neonatos nacidos en el Hospital Regional de Encarnación, Itapúa- Paraguay. Material y Método: Estudio prospectivo de casos y

Esta investigación se realizó en el marco de la convocatoria 2006 para proyectos de investigación de la Dirección General de Investigaciones Científicas y Tecnológicas de la Universidad Nacional de Asunción.

Correspondencia a:

E-mail: rcorvalan@clik.com.py 
controles de marzo/2006 a febrero/2007. Se consideró caso a todo neonato con malformación congénita, y control a todo niño sano del mismo sexo que naciera inmediatamente después. No se incluyeron los nacimientos ocurridos fuera del hospital. Se consideró exposición a cualquier contacto con agroquímicos, así como a otros factores de riesgo conocidos para malformación congénita. Resultados: Se analizaron 52 casos y 87 controles. El promedio de nacimientos por mes fue de 216 . Los factores de riesgo asociados significativamente fueron: vivir cerca de campos fumigados (OR 2,46 IC95\% 1,09 - 5,57, p < 0,02), vivienda ubicada a $<1 \mathrm{~km}(\mathrm{OR}=2,66$ IC 95\% 1,19 - 5,97), $\mathrm{p}<0,008)$, almacenamiento de plaguicidas en el hogar $(\mathrm{OR}$ 15,35 IC95\% 1,96 - 701,63 p < 0,003), contacto en forma directa o accidental con plaguicidas(OR 3,19 IC95\% 0,97-11,4, p < 0,04), antecedente de malformación en la familia (OR 6,81 IC5\% 1,94 - 30,56, p < 0,001). Los demás factores de riesgo conocidos para malformaciones no tuvieron significancia estadística. Conclusión: Los resultados muestran una asociación entre exposición a pesticidas y malformaciones congénitas. Se requiere de estudios futuros para confirmar estos hallazgos.

(Palabras clave: Malformaciones congénitas, agrotóxicos, exposición a plaguicidas).

Pediatr. (Asunción), Vol. 34; No 2; 2007

ESTE TRABAJO LO PUEDE ENCONTRAR EN EXTENSO EN WWW.SCIELO.CL 